\title{
A representation for Green's function retrieval by multidimensional deconvolution
}

\author{
Kees Wapenaar ${ }^{\text {a) }}$ and Joost van der Neut \\ Department of Geotechnology, Delft University of Technology, P.O. Box 5048, 2600 GA Delft, The Netherlands \\ c.p.a.wapenaar@tudelft.nl, j.r.vanderneut@tudelft.nl
}

\begin{abstract}
Green's function retrieval by crosscorrelation may suffer from irregularities in the source distribution, asymmetric illumination, intrinsic losses, etc. Multidimensional deconvolution (MDD) may overcome these limitations. A unified representation for Green's function retrieval by MDD is proposed. From this representation, it follows that the traditional crosscorrelation method gives a Green's function of which the source is smeared in space and time. This smearing is quantified by a space-time point-spread function (PSF), which can be retrieved from measurements at an array of receivers. MDD removes this PSF and thus deblurs and deghosts the source of the Green's function obtained by correlation.

(C) 2010 Acoustical Society of America

PACS numbers: 43.60.Ac, 43.60.Tj [CG]

Date Received: October 2, 2010 Date Accepted: October 12, 2010
\end{abstract}

\section{Introduction}

Research in the past decennium has revealed that the crosscorrelation of recordings of a wave field at two receivers yields, under specific conditions, the Green's function between these receivers. ${ }^{1,2}$ This has led to numerous applications in the fields of ultrasonics, ${ }^{3}$ regional seismology, ${ }^{4}$ exploration seismics, ${ }^{5,6}$ underwater acoustics, ${ }^{7}$ medical imaging, ${ }^{8}$ and infrasonic investigations of the atmosphere. ${ }^{9}$ For open systems, the main assumptions are that the medium is lossless and that the wave field is generated by a regular distribution of sequential transient sources or uncorrelated noise sources, enclosing the receivers. Heuristic arguments and practical results show that reasonably accurate Green's functions can be retrieved, even when these assumptions are not entirely fulfilled. However, when the underlying assumptions are severely violated, the retrieved Green's functions are inaccurate and contaminated with artifacts.

Recently, researchers have shown that some of the assumptions can be circumvented when the correlation is replaced by deconvolution. ${ }^{5,10-13}$ The proposed approaches vary from case to case: Some employ a trace-by-trace spectral whitening ${ }^{10}$ or deconvolution; ${ }^{11}$ others account for the three-dimensional (3D) character of the field; $5,12,13$ and some are designed for transient sources ${ }^{5,12}$ and others for noise sources. ${ }^{10,11,13}$ Here we derive a unified representation for Green's function retrieval by multidimensional deconvolution (MDD), which applies among others to 3D diffusion and wave phenomena from transient or noise sources.

\section{Basic Green's function representation}

We consider a Green's function $G\left(\mathbf{x}, \mathbf{x}_{S}, t\right)$ obeying the equation

$$
\left[\sum_{n} a_{n}(\mathbf{x}, t) * \frac{\partial^{n}}{\partial t^{n}}-H(\mathbf{x})\right] G\left(\mathbf{x}, \mathbf{x}_{S}, t\right)=\delta\left(\mathbf{x}-\mathbf{x}_{S}\right) \delta(t),
$$

where $\mathbf{x}_{S}$ is the source position, the asterisk $(*)$ denotes temporal convolution, the $a_{n}(\mathbf{x}, t)$ are medium parameters, and $H(\mathbf{x})$ is a spatial differential operator. ${ }^{14}$ For example, for

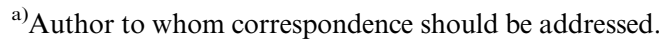


diffusion phenomena, $a_{1}(\mathbf{x}, t)=\delta(t)$ [note that $\delta(t) * \partial G / \partial t=\partial G / \partial t$ ], all other $a_{n}$ are zero, and $H(\mathbf{x})=\nabla \cdot D \nabla$, where $D(\mathbf{x})$ is the diffusion coefficient. For acoustic waves in media with losses, only the $a_{1}$ and $a_{2}$ are non-zero and $H(\mathbf{x})=\nabla \cdot \rho^{-1} \nabla$, where $\rho(\mathbf{x})$ is the mass density; for the special case of a lossless medium $a_{1}=0$ and $a_{2}=\delta(t) / \rho c^{2}$, with $c(\mathbf{x})$ as the propagation velocity. In these examples, $H$ is a symmetric operator, which implies $G\left(\mathbf{x}, \mathbf{x}_{S}, t\right)=G\left(\mathbf{x}_{S}, \mathbf{x}, t\right)$.

In 3D space we define a volume $\mathbb{V}$ bounded by a surface $\mathbb{S}$ with outward pointing normal vector $\mathbf{n}$. We introduce the bilinear form $L(f, g)$ via $^{14}$

$$
\int_{\mathbb{V}}[f *(H g)-(H f) * g] \mathrm{d} \mathbb{V}=\oint_{\mathbb{S}} L(f, g) \mathrm{d} \mathbb{S},
$$

where $f$ and $g$ are space- and time-dependent functions. For example, for $H(\mathbf{x})=\nabla \cdot b \nabla$ (where, e.g., $b=D$ or $b=1 / \rho$ ) we obtain, using the theorem of Gauss,

$$
L(f, g)=b(f * \nabla g-\nabla f * g) \cdot \mathbf{n} .
$$

We define a reference Green's function $\bar{G}\left(\mathbf{x}, \mathbf{x}_{R}, t\right)$ (the bar denotes the reference situation) obeying Eq. (1), but with the source term on the right-hand side replaced by $\delta\left(\mathbf{x}-\mathbf{x}_{R}\right) \delta(t)$. In $\mathbb{V}$ the $a_{n}$ and $H$ for $\bar{G}$ are the same as for $G$ in Eq. (1), but they may be different outside $\mathbb{V}$. We take the reference medium for $\bar{G}$ homogeneous outside $\mathbb{V}$. Moreover, we choose $\mathbf{x}_{R}$ inside $\mathbb{V}$ and $\mathbf{x}_{S}$ outside $\mathbb{V}$. Figure 1(a) shows $G\left(\mathbf{x}_{R}, \mathbf{x}_{S}, t\right)$ and $G\left(\mathbf{x}, \mathbf{x}_{S}, t\right)$ (with $\mathbf{x}$ on $\mathbb{S}$ ) in an arbitrary inhomogeneous medium (the black dots denote scatterers) and Fig. 1(b) shows $\bar{G}\left(\mathbf{x}, \mathbf{x}_{R}, t\right)=\bar{G}\left(\mathbf{x}_{R}, \mathbf{x}, t\right)$ in the reference medium.

We derive a convolution-type representation for the field at $\mathbf{x}_{R}$. To this end we convolve both sides of Eq. (1) with $\bar{G}\left(\mathbf{x}, \mathbf{x}_{R}, t\right)$. Similarly, we convolve both sides of the equation for $\bar{G}\left(\mathbf{x}, \mathbf{x}_{R}, t\right)$ with $G\left(\mathbf{x}, \mathbf{x}_{S}, t\right)$. Subtracting the two equations, integrating both sides over $\mathbb{V}$, using Eq. (2) and the property $f * \partial^{n} g / \partial t^{n}=g * \partial^{n} f / \partial t^{n}$ yields

$$
G\left(\mathbf{x}_{R}, \mathbf{x}_{S}, t\right)=\oint_{\mathbb{S}} L\left(\bar{G}\left(\mathbf{x}, \mathbf{x}_{R}, t\right), G\left(\mathbf{x}, \mathbf{x}_{S}, t\right)\right) \mathrm{d} \mathbb{S} .
$$

This representation is the starting point for Green's function retrieval by MDD.

\section{Simplification of the integral}

From here onward we choose $H(\mathbf{x})=\nabla \cdot b \nabla$. Hence, using Eq. (3), Eq. (4) becomes

$$
G\left(\mathbf{x}_{R}, \mathbf{x}_{S}, t\right)=\oint_{\mathbb{S}} b(\mathbf{x})\left\{\bar{G}\left(\mathbf{x}, \mathbf{x}_{R}, t\right) * \nabla G\left(\mathbf{x}, \mathbf{x}_{S}, t\right)-\nabla \bar{G}\left(\mathbf{x}, \mathbf{x}_{R}, t\right) * G\left(\mathbf{x}, \mathbf{x}_{S}, t\right)\right\} \cdot \mathbf{n d} \mathbb{S} .
$$

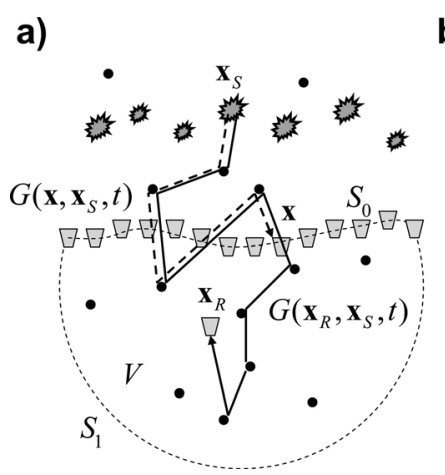

b)

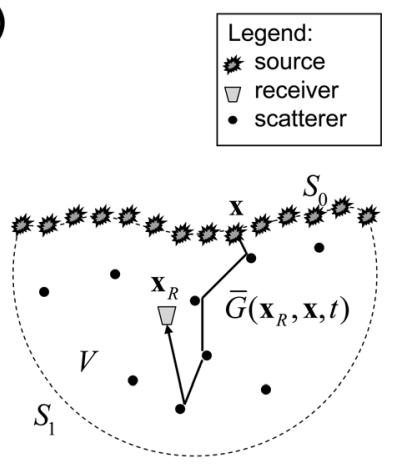

Fig. 1. Green's functions in an arbitrary inhomogeneous open system. (a) Actual situation. These Green's functions represent the observations at $\mathbf{x}_{R}$ and $\mathbf{x}$. (b) Reference situation. This Green's function is the response of a virtual source at $\mathbf{x}$. MDD resolves this Green's function from the observations at $\mathbf{x}_{R}$ and $\mathbf{x}$. 
As indicated in Fig. 1, $\mathbb{S}$ consists of an open surface $\mathbb{S}_{0}$ and a half-sphere $\mathbb{S}_{1}$. By letting the radius of the half-sphere go to infinity, the integral along $\mathbb{S}_{1}$ vanishes (Sommerfeld radiation condition). Hence, the integration in Eq. (5) can be restricted to the open boundary $\mathbb{S}_{0}$. On $\mathbb{S}_{0}$, we write $G\left(\mathbf{x}, \mathbf{x}_{S}, t\right)$ as the superposition of inward (into $\mathbb{V}$ ) and outward (away from $\mathbb{V}$ ) propagating fields, according to $G=G^{\text {in }}+G^{\text {out }}$. Note that the reference Green's function $\bar{G}\left(\mathbf{x}, \mathbf{x}_{R}, t\right)$ is outward propagating at $\mathbb{S}_{0}$. By using pseudo-differential operator theory, ${ }^{15}$ it follows that the two terms in $\int b\left\{\bar{G} * \nabla G^{\text {out }}-\nabla G * G^{\text {out }}\right\} \cdot \mathbf{n d} \mathbb{S}_{0}$ cancel each other, whereas the terms in $\int b\left\{\bar{G} * \nabla G^{\text {in }}-\nabla \bar{G} * G^{\text {in }}\right\} \cdot \mathbf{n d} \mathbb{S}_{0}$ are identical but opposite in sign. (This can be more easily derived with a far-field approximation, ${ }^{16}$ but the pseudo-differential approach circumvents this approximation.) Using this in Eq. (5), together with $\bar{G}\left(\mathbf{x}, \mathbf{x}_{R}, t\right)=\bar{G}\left(\mathbf{x}_{R}, \mathbf{x}, t\right)$, we obtain

$$
G\left(\mathbf{x}_{R}, \mathbf{x}_{S}, t\right)=\int_{\mathbb{S}_{0}} \bar{G}_{d}\left(\mathbf{x}_{R}, \mathbf{x}, t\right) * G^{\text {in }}\left(\mathbf{x}, \mathbf{x}_{S}, t\right) \mathrm{d} \mathbb{S}_{0},
$$

where $\bar{G}_{d}\left(\mathbf{x}_{R}, \mathbf{x}, t\right)=-2 b(\mathbf{x}) \mathbf{n} \cdot \nabla \bar{G}\left(\mathbf{x}_{R}, \mathbf{x}, t\right)$. The subscript " $d$ " denotes that the source at $\mathbf{x}$ has a dipole character because of the operator $\mathbf{n} \cdot \nabla$. Note that $\bar{G}_{d}\left(\mathbf{x}_{R}, \mathbf{x}, t\right)$ is the response to be resolved from Eq. (6). If representation (6) was a single equation, the inverse problem would be ill-posed. However, Eq. (6) exists for each source position $\mathbf{x}_{S}$, which we will denote from hereon by $\mathbf{x}_{S}^{(i)}$, where $i$ denotes the source number. Solving this ensemble of equations for $\bar{G}_{d}\left(\mathbf{x}_{R}, \mathbf{x}, t\right)$ involves MDD. Recall that the bar denotes a reference situation which is equal to the actual medium in $\mathbb{V}$ but homogeneous outside $\mathbb{V}$. This means that the retrieved Green's function $\bar{G}_{d}\left(\mathbf{x}_{R}, \mathbf{x}, t\right)$ is correct for the medium in $\mathbb{V}$ but does not include scattering from outside $\mathbb{V}$, see Fig. 1(b). In the example in Sec. 4 we show that this may improve the interpretability of the retrieved Green's function. The reference medium does not restrict the method, because the "measured" Green's functions $G$ and $G^{\text {in }}$ in Eq. (6) are defined in the actual medium, see Fig. 1(a).

\section{Transient sources}

To comply with practical situations, the Green's functions $G$ and $G^{\text {in }}$ in representation (6) should be replaced by responses of real sources, i.e., Green's functions convolved with source functions. For the responses of transient sources we write $u^{\text {in }}\left(\mathbf{x}, \mathbf{x}_{S}^{(i)}, t\right)$ $=G^{\text {in }}\left(\mathbf{x}, \mathbf{x}_{S}^{(i)}, t\right) * s^{(i)}(t)$ and $u\left(\mathbf{x}_{R}, \mathbf{x}_{S}^{(i)}, t\right)=G\left(\mathbf{x}_{R}, \mathbf{x}_{S}^{(i)}, t\right) * s^{(i)}(t)$. Convolving both sides of Eq. (6) with $s^{(i)}(t)$, we thus obtain

$$
u\left(\mathbf{x}_{R}, \mathbf{x}_{S}^{(i)}, t\right)=\int_{\mathbb{S}_{0}} \bar{G}_{d}\left(\mathbf{x}_{R}, \mathbf{x}, t\right) * u^{\mathrm{in}}\left(\mathbf{x}, \mathbf{x}_{S}^{(i)}, t\right) \mathrm{d} \mathbb{S}_{0} .
$$

Solving Eq. (7) in a least-squares sense is equivalent to solving its normal equation. We obtain the normal equation by crosscorrelating both sides of Eq. (7) with $u^{\text {in }}\left(\mathbf{x}^{\prime}, \mathbf{x}_{S}^{(i)}, t\right)$ (with $\mathbf{x}^{\prime}$ on $\mathbb{S}_{0}$ ) and taking the sum over all sources. ${ }^{13}$ This gives

$$
C\left(\mathbf{x}_{R}, \mathbf{x}^{\prime}, t\right)=\int_{\mathbb{S}_{0}} \bar{G}_{d}\left(\mathbf{x}_{R}, \mathbf{x}, t\right) * \Gamma\left(\mathbf{x}, \mathbf{x}^{\prime}, t\right) \mathrm{d} \mathbb{S}_{0}
$$

where

$$
\begin{gathered}
C\left(\mathbf{x}_{R}, \mathbf{x}^{\prime}, t\right)=\sum_{i} u\left(\mathbf{x}_{R}, \mathbf{x}_{S}^{(i)}, t\right) * u^{\text {in }}\left(\mathbf{x}^{\prime}, \mathbf{x}_{S}^{(i)},-t\right), \\
\Gamma\left(\mathbf{x}, \mathbf{x}^{\prime}, t\right)=\sum_{i} u^{\text {in }}\left(\mathbf{x}, \mathbf{x}_{S}^{(i)}, t\right) * u^{\text {in }}\left(\mathbf{x}^{\prime}, \mathbf{x}_{S}^{(i)},-t\right) .
\end{gathered}
$$

Equation (9), defining the correlation function, $C\left(\mathbf{x}_{R}, \mathbf{x}^{\prime}, t\right)$, is the traditional expression used for Green's function retrieval by crosscorrelation for the situation of transient 
sources ${ }^{5,6}$ (except that instead of $u^{\text {in }}$ one usually takes a time-gated version of the total field $u$ ). Equation (8) shows that this correlation function is proportional to the Green's function $\bar{G}_{d}\left(\mathbf{x}_{R}, \mathbf{x}, t\right)$ with its source smeared in space and time by $\Gamma\left(\mathbf{x}, \mathbf{x}^{\prime}, t\right)$. We call $\Gamma\left(\mathbf{x}, \mathbf{x}^{\prime}, t\right)$ the space-time point-spread function (PSF). If we would have a regular distribution of impulsive sources $\mathbf{x}_{S}^{(i)}$ along a sufficiently large source boundary, and if the actual medium outside $\mathbb{V}$ were homogeneous and lossless, the PSF as defined in Eq. (10) would approach a band-limited delta function. In practice, there are many factors that make the PSF deviate from a delta function. Among these factors are the irregularity of the source distribution, medium inhomogeneities, a finite aperture, asymmetric illumination, multiple reflections in the illuminating wave field, intrinsic losses, etc. In all those cases Eq. (8) needs to be inverted by MDD, i.e., the effects of the PSF $\Gamma\left(\mathbf{x}, \mathbf{x}^{\prime}, t\right)$ need to be removed from the correlation function $C\left(\mathbf{x}_{R}, \mathbf{x}^{\prime}, t\right)$ to obtain the Green's function $\bar{G}_{d}\left(\mathbf{x}_{R}, \mathbf{x}, t\right)$. This is illustrated with a numerical example in Fig. 2.

\section{Noise sources}

We show that Eq. (8) also holds for the situation of simultaneously acting uncorrelated noise sources. To this end, we define the correlation function and the PSF, respectively, as

$$
C\left(\mathbf{x}_{R}, \mathbf{x}^{\prime}, t\right)=\left\langle u\left(\mathbf{x}_{R}, t\right) * u^{\text {in }}\left(\mathbf{x}^{\prime},-t\right)\right\rangle
$$
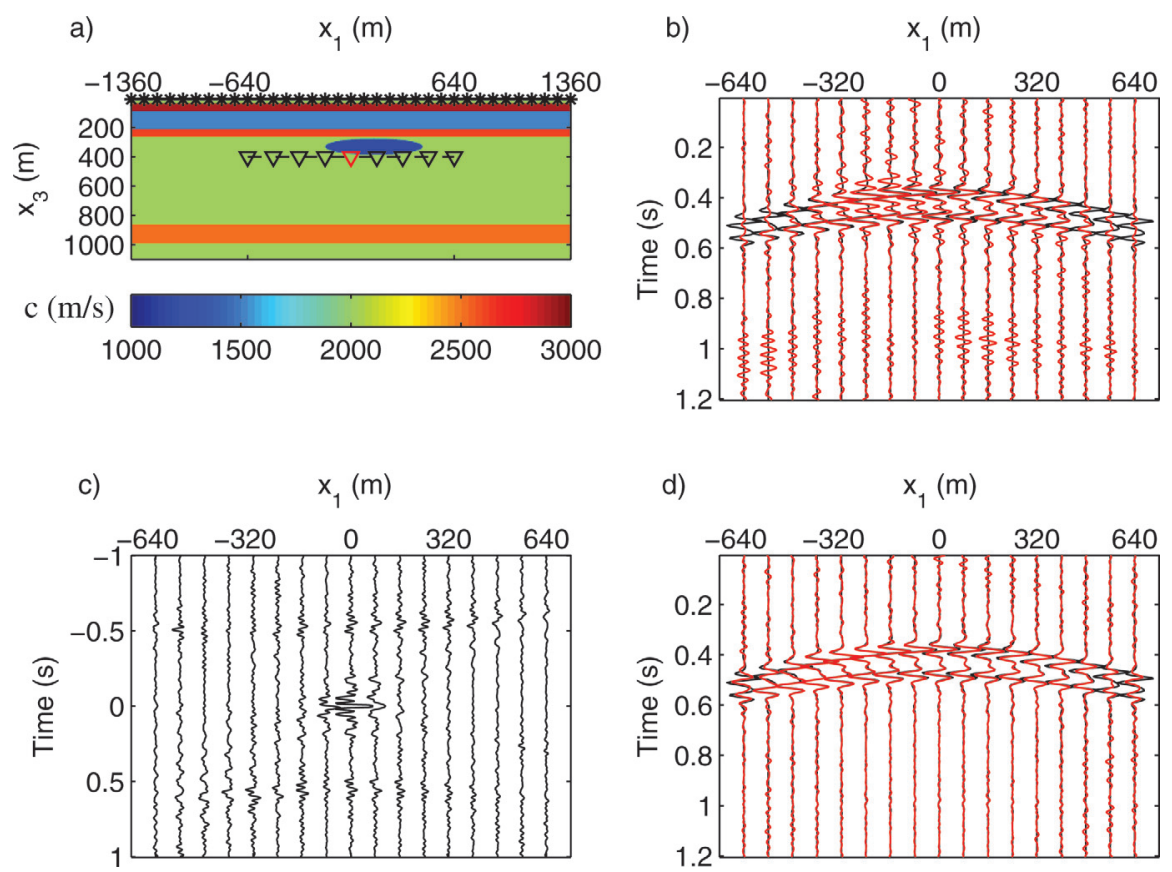

Fig. 2. Numerical example. (a) Seismic exploration configuration with sources at the surface (stars) and receivers in a horizontal borehole (triangles). The red triangle indicates the virtual source. The blue ellipse represents a gas cloud which acts as an acoustic lens. The deep orange layer represents a hydrocarbon reservoir. (b) Red traces: Virtual source response $C\left(\mathbf{x}_{R}, \mathbf{x}^{\prime}, t\right)$ obtained with the correlation method [Eq. (9), but with decomposed wave fields at both receivers ${ }^{17}$ ], with $\mathbf{x}^{\prime}$ being the virtual source position and $\mathbf{x}_{R}$ varying along the borehole. Black traces: Directly modeled reflection response of the reservoir. The match is reasonable, but we also observe distortions along the reflection event as well as severe ghost events. (c) PSF $\Gamma\left(\mathbf{x}, \mathbf{x}^{\prime}, t\right)$ (clipped at $50 \%$ of its maximum amplitude). (d) Red traces: Virtual source response $\bar{G}_{d}\left(\mathbf{x}_{R}, \mathbf{x}, t\right)$ obtained by MDD, i.e., by deconvolving the correlation function in (b) by the PSF in (c). Black traces: Directly modeled reflection response of the reservoir. Note that the match is very good. Moreover, the retrieved reflection response is free of multiple scattering caused by the earth's surface, the geological layers, and the gas cloud. This response can subsequently be used for imaging and characterization of the reservoir layer. 


$$
\Gamma\left(\mathbf{x}, \mathbf{x}^{\prime}, t\right)=\left\langle u^{\text {in }}(\mathbf{x}, t) * u^{\text {in }}\left(\mathbf{x}^{\prime},-t\right)\right\rangle,
$$

where $\langle\cdot\rangle$ denotes ensemble averaging, which is in practice replaced by integration over sufficiently long time and/or averaging over different time intervals. The noise responses in Eqs. (11) and (12) are defined as $u^{\text {in }}\left(\mathbf{x}^{\prime}, t\right)=\sum_{i} G^{\text {in }}\left(\mathbf{x}^{\prime}, \mathbf{x}_{S}^{(i)}, t\right) * N^{(i)}(t)$ and $u\left(\mathbf{x}_{R}, t\right)$ $=\sum_{j} G\left(\mathbf{x}_{R}, \mathbf{x}_{S}^{(j)}, t\right) * N^{(j)}(t)$, in which the noise signals are mutually uncorrelated, according to $\left\langle N^{(j)}(t) * N^{(i)}(-t)\right\rangle=\delta_{i j} S^{(i)}(t)$. Upon substitution into Eqs. (11) and (12) it follows that the correlation function and the PSF as defined in Eqs. (11) and (12) are identical to those defined in Eqs. (9) and (10), assuming $s^{(i)}(t) * s^{(i)}(-t)=S^{(i)}(t)$. Hence, whether we consider transient or noise sources, Eq. (8) is the relation that needs to be inverted by MDD to resolve the Green's function $\bar{G}_{d}\left(\mathbf{x}_{R}, \mathbf{x}, t\right)$.

\section{Discussion and conclusions}

The methodology of retrieving the Green's function by crosscorrelation has a number of attractive properties as well as several limitations. The main attractiveness of the method is that a virtual source response can be obtained by crosscorrelating responses at two receivers, without the need to know the medium parameters and the sources. For open systems the method works well when the receivers that are used in the correlation process are surrounded by a regular distribution of sequential transient sources or uncorrelated noise sources with equal autocorrelation functions. However, in practical situations the method may suffer from irregularities in the source distribution, asymmetric illumination, intrinsic losses, etc.

We have shown that these imperfections manifest themselves as a blurring of the source of the Green's function in the spatial direction and the generation of ghosts in the temporal direction. These effects are quantified as a convolution of the Green's function with the space-time PSF [Eq. (8)]. As such, our PSF plays a similar role as the spatial PSF in optical, acoustic, and seismic imaging systems. ${ }^{18-20}$ When the PSF of an imaging system is known, the resolution of an image can be improved by deconvolving for the PSF. ${ }^{21}$ In a similar way, the blurred source-with-ghosts of the Green's function obtained by the crosscorrelation method can be deblurred and deghosted by deconvolving for the space-time PSF, i.e., by inverting Eq. (8) by MDD. We have demonstrated this with a numerical example for seismic exploration data with transient sources (Fig. 2), but similar improvements can be achieved for Green's function retrieval from ambient noise. ${ }^{13}$

Of course also the MDD method has its limitations. First, in order to retrieve the Green's function $\bar{G}_{d}\left(\mathbf{x}_{R}, \mathbf{x}, t\right)$ it does not suffice to have two receivers only, at $\mathbf{x}_{R}$ and $\mathbf{x}$, because $\mathbf{x}$ is assumed to be an element of a regular (or regularized) array of receivers along $\mathbb{S}_{0}$, see Fig. 1(a). Second, the expressions for the correlation function [Eqs. (9) and (11)] and for the PSF [Eqs. (10) and (12)] contain the inward propagating field $u^{\text {in }}$ on $\mathbb{S}_{0}$ (propagating into $\mathbb{V}$ ). In order to extract this inward propagating field from the total field, either multicomponent receiver ${ }^{22}$ or two receiver surfaces close to each other ${ }^{23}$ are required. Alternatively, when multiple scattering is weak, one could correlate the total fields and extract the PSF by applying a time window around $t=0$. Third, MDD involves an inversion of an integral equation, which in practice is achieved by matrix inversion. Apart from the higher cost (in comparison with the correlation method), this matrix inversion can become unstable when there are gaps in the illumination directions of the virtual source. Despite these limitations, for applications in which the data are measured with arrays of receivers, Green's function retrieval by MDD has the potential to obtain virtual sources that are better focused and contain less ghost events than those obtained by the crosscorrelation method.

During the review process it has been pointed out that there is a link with an interesting method called "time exposure acoustics." 24 This method has been designed to image scatterers that are illuminated by ambient noise. Assuming these scatterers can be seen as uncorrelated noise sources (meaning their mutual distance should be larger than the spatial correlation length of the noise), they can be imaged by 
backpropagating the recorded noise. The image quality improves with increasing exposure time. The optimum achievable image is quantified by a spatial convolution of the scattering model with a noise-derived PSF. There are also important differences. Time exposure acoustics is model-driven (it uses a background velocity for the backpropagation and for the computation of the PSF), and it ignores multiple scattering. Green's function retrieval by MDD is data-driven (the correlation function and the PSF are obtained from measured responses), it does not assume a minimum distance between scatterers, and it accounts for multiple scattering. The output is not an image but a virtual source response that can be used for further processing (tomographic inversion, model-driven imaging, etc.).

\section{References}

${ }^{1}$ R. L. Weaver and O. I. Lobkis, "Ultrasonics without a source: Thermal fluctuation correlations at $\mathrm{MHz}$ frequencies," Phys. Rev. Lett. 87, 134301 (2001).

${ }^{2} \mathrm{R}$. Snieder, "Extracting the Green's function from the correlation of coda waves: A derivation based on stationary phase," Phys. Rev. E 69, 046610 (2004).

${ }^{3}$ O. I. Lobkis and R. L. Weaver, "On the emergence of the Green's function in the correlations of a diffuse field," J. Acoust. Soc. Am. 110, 3011-3017 (2001).

${ }^{4}$ N. M. Shapiro and M. Campillo, "Emergence of broadband Rayleigh waves from correlations of the ambient seismic noise," Geophys. Res. Lett. 31, L07614 (2004).

${ }^{5} \mathrm{G}$. T. Schuster and M. Zhou, "A theoretical overview of model-based and correlation-based redatuming methods," Geophysics 71, SI103-SI110 (2006).

${ }^{6} \mathrm{~A}$. Bakulin and R. Calvert, "The virtual source method: Theory and case study," Geophysics 71, SI139SI150 (2006).

${ }^{7}$ P. Roux and M. Fink, "Green's function estimation using secondary sources in a shallow water environment," J. Acoust. Soc. Am. 113, 1406-1416 (2003).

${ }^{8}$ K. G. Sabra, S. Conti, P. Roux, and W. A. Kuperman, "Passive in vivo elastography from skeletal muscle noise," Appl. Phys. Lett. 90, 194101 (2007).

${ }^{9}$ M. M. Haney, "Infrasonic ambient noise interferometry from correlations of micro-baroms," Geophys. Res. Lett. 36, L19808 (2009).

${ }^{10}$ G. D. Bensen, M. H. Ritzwoller, M. P. Barmin, A. L. Levshin, F. Lin, M. P. Moschetti, N. M. Shapiro, and Y. Yang, "Processing seismic ambient noise data to obtain reliable broad-band surface wave dispersion measurements," Geophys. J. Int. 169, 1239-1260 (2007).

${ }^{11}$ R. Snieder, J. Sheiman, and R. Calvert, "Equivalence of the virtual-source method and wave-field deconvolution in seismic interferometry," Phys. Rev. E 73, 066620 (2006).

${ }^{12} \mathrm{~K}$. Wapenaar, J. van der Neut, and E. Ruigrok, "Passive seismic interferometry by multidimensional deconvolution," Geophysics 73, A51-A56 (2008).

${ }^{13}$ J. van der Neut, E. Ruigrok, D. Draganov, and K. Wapenaar, "Retrieving the earth's reflection response by multi-dimensional deconvolution of ambient seismic noise," in EAGE, Extended Abstracts, Session: P406 (2010).

${ }^{14}$ R. Snieder, K. Wapenaar, and U. Wegler, “Unified Green's function retrieval by cross-correlation; Connection with energy principles,” Phys. Rev. E 75, 036103 (2007).

${ }^{15}$ L. Fishman, "One-way propagation methods in direct and inverse scalar wave propagation modeling," Radio Sci. 28, 865-876 (1993).

${ }^{16} \mathrm{~K}$. Wapenaar, J. Fokkema, and R. Snieder, "Retrieving the Green's function in an open system by crosscorrelation: A comparison of approaches (L)," J. Acoust. Soc. Am. 118, 2783-2786 (2005).

${ }^{17}$ K. Mehta, A. Bakulin, J. Sheiman, R. Calvert, and R. Snieder, "Improving the virtual source method by wavefield separation," Geophysics 72, V79-V86 (2007).

${ }^{18}$ M. Born and E. Wolf, Principles of Optics (Pergamon Press, London, 1965).

${ }^{19}$ S. J. Norton, “Annular array imaging with full-aperture resolution,” J. Acoust. Soc. Am. 92, 3202-3206 (1992).

${ }^{20} \mathrm{G}$. T. Schuster and J. Hu, "Green's function for migration: Continuous recording geometry," Geophysics 65, 167-175 (2000).

${ }^{21}$ P. Jansson, Deconvolution of Images and Spectra (Academic Press, San Diego, 1997).

${ }^{22}$ L. Amundsen and A. Reitan, "Decomposition of multicomponent sea-floor data into up-going and downgoing P- and S-waves," Geophysics 60, 563-572 (1995).

${ }^{23}$ D. Hill, L. Combee, and J. Bacon, "Over/under acquisition and data processing: The next quantum leap in seismic technology?" First Break 24, 81-95 (2006).

${ }^{24}$ S. J. Norton and I. J. Won, “Time exposure acoustics,” IEEE Trans. Geosci. Remote Sens. 38, 1337 1343 (2000). 